\title{
Surface-based Internal Multiple Elimination in the CMP Domain - Theory and Application Strategies on Land Seismic Data
}

\author{
Shiguang DENG, Wenjin ZHAO, and Zhiwei LIU \\ Chinese Academy of Geological Sciences, Beijing, People's Repulic of China; \\ e-mails: dengshiguang@live.com,zhaowj@cae.cn, \\ zwliu007@sina.com (corresponding author)
}

\section{Abstract}

The data-driven internal multiple elimination (IME) method based on feedback model, which includes CFP-based, surface-based and inversion-based methods, are successfully applied to marine datasets. However, these methods are computationally expensive and not always straightforward on land datasets. In this paper, we first proved that the surface-based IME method, which is the most computationally efficient method among the three methods, can be derived from the CFP theory. Then we extend it to CMP domain under the assumption of locally lateral invariance of the earth, which makes it more computationally efficient. In addition, we proposed applying a time-variant taper based on the first Fresnel zone to predict the multiples more percisely. Besides, the improved $\mathrm{S} / \mathrm{N}$ ratio and dense offset distribution can be obtained by using the CMP supergather, which makes the CMP-oriented method more suitable for land data. Some practical processing strategies are proposed via case study. The effectiveness of the proposed method is demonstrated with the application to synthetic and field data.

Key words: internal multiple, feedback model, surface-based, CMPoriented, land seismic data. 


\section{INTRODUCTION}

Multiple reflections affect seismic imaging quality, especially when strong subsurface reflectors exist. The interference of multiple energy with primary events could result in interpretation uncertainties. It is necessary to remove multiples before subsequent processing. In general, multiples consist of freesurface multiples and internal multiples. Free-surface multiples are multiples that have experienced at least one downward reflection at the air-water "freesurface"; internal multiples are multiples that have all of their downward reflections below the free surface. Land seismic data is mainly affected by internal multiples. Internal multiples have experienced reflectors that are in general more remote and harder to precisely define (in comparison with freesurface multiples); hence, internal multiples are more difficult to predict and attenuate (Weglein 1999).

Two major internal multiple elimination (IME) methods, based on wave theory, are the inverse-scattering series (ISS) and feedback methods. The inverse scattering series (ISS) method for internal multiple elimination is discussed specifically by Araujo et al. (1994), Coates and Weglein (1996), Weglein et al. (1997). The ISS method is fully data-driven and does not require any subsurface information. However, the cost of the ISS approach is considerably greater than the feedback method (Verschuur and Prein 1999). In practice, the feedback method would be a more effective choice. Berkhout and Verschuur firstly proposed the feedback method for the surface-related multiple elimination (SRME) (Berkhout 1982, Verschuur 1991, Verschuur et al. 1992, Berkhout and Verschuur 1997, Verschuur and Berkhout 1997). Berkhout and Verschuur (1997) extended the algorithm from surface to internal multiples by replacing shot records with common-focus-point (CFP) gathers (Berkhout 1997, Thorbecke 1997). Berkhout and Verschuur (2005) illustrated the internal-multiple-removal algorithm with numerical examples. This algorithm can be formulated in terms of boundary-related and layerrelated versions. Verschuur and Berkhout (2005) demonstrated the strategy for applying the two versions of internal-multiple-removal algorithm on physical-model and field data. The boundary-related approach requires the construction of CFP gathers, using focusing operators with correct traveltimes, while the layer-related approach allows traveltime errors. From a cost perspective, the layer-related method costs twice as much computation time as the boundary-related method, even though the boundary-related method involves more user interactions. Despite the extra calculation cost, the ease of use and the robustness of the layer-related approach make it preferable to the boundary-related approach in most situations. Jakubowicz (1998) proposed the surface-based IME method, in which the need for CFP gathers in the boundary-related method is avoided, and internal multiples can 
be estimated directly from the data measured on the surface. In van Borselen (2002), an extension of this procedure is illustrated to remove internal multiples that have crossed a pseudo-boundary that is chosen to lie between two internal reflectors. Thus, the data-driven internal multiple elimination based on surface data can be applied either in boundary-related version or layerrelated version. Recently, Ypma and Verschuur (2013) redefined internal multiple elimination as a full waveform inversion process, following the principles of estimating primaries by sparse inversion (EPSI) (van Groenestijn and Verschuur 2009a,b). Song et al. (2013) compared three feedback IME methods: the CFP-based method, the surface-based method and the inversion-based method. Internal multiples estimated by the inversion-based generalized EPSI method are clearer and more spatially continuous. However, from a cost perspective, the surface-based method is more computationally efficient. The CFP-based method is twice expensive as the surface-based method, and the generalized EPSI is several tens of times more expensive than the surface-based method.

For land data, internal multiple elimination is more difficult than in the marine case. Complex near-surface condition, irregular geometry patterns, poor $\mathrm{S} / \mathrm{N}$ ratios and source/receiver coupling issues, are key obstacles that deteriorate the performance of multiple attenuation algorithms (Kelamis et al. 2006, Luo et al. 2011). In this situation, the multiple elimination method in shot domain becomes cumbersome. An alternative choice is to eliminate the internal multiples in the CMP domain if the approximation of a lowrelief structure is valid. Yuan et al. (2009) described a processing strategy which combines stacking and $f-k$ filtering in CMP domain for multiple elimination on land seismic data. Kelamis et al. (2002) and Alá'i and Verschuur (2006) apply the CFP-based method in CMP domain for the internal multiple elimination on land data. For the feedback model IME methods, trace interpolation and offset regulation are requried. Such a processing can be more suitable to perform in CMP domain. With the use of CMP supergather, the $\mathrm{S} / \mathrm{N}$ ratios and spatial sampling density can be improved considerably. In addition, the CMP-oriented method can be applied to both 2D and 3D data sets.

In this paper, we first derived the surface-based IME method based on the CFP theory, showing the relationship between CFP-based and surfacebased IME methods. Then we extend the surface-based IME method to CMP domain under the assumption of locally lateral invariance of the earth, which makes it more computationally efficient. In addition, we proposed, by applying a time-variant taper based on the first Fresnel zone, to predict the multiples more percisely. The effectiveness of the proposed CMP-oriented method is demonstrated with synthetic and field land data. Finally, we end up with some discussion and conclusions. 


\section{THEORY AND METHODOLOGY}

Using the detail-hiding operator notation (Berkhout 1982), which uses data matrix (Fig. 1) to represent discrete prestack data volumes for a single frequency, the surface-related multiple prediction can be formulated as

$$
\mathbf{M}=A(f) \mathbf{P}_{0} \mathbf{P},
$$

where $\mathbf{M}$ represents the predicted surface-related multiple, $\mathrm{A}(f)$ compensates for source and detector properties. $\mathbf{P}_{0}$ represents the data without surfacerelated multiples and $\mathbf{P}$ represents the data with surface-related multiples. Figure 2 illustrates the process of the surface-related multiple prediction in $t$ $x$ domain. The multiple removal process can be written in an iterative manner (Berkhout and Verschuur 1997):

$$
\mathbf{P}_{0}^{(\mathrm{i}+1)}=\mathbf{P}-A(f) \mathbf{P}_{0}^{(\mathrm{i})} \mathbf{P}
$$

with $\mathbf{P}_{0}^{(\mathrm{i}+1)}$ being the estimated primaries in the $(\mathrm{i}+1)$-th iteration and initial estimate $\mathbf{P}_{0}^{(0)}=\mathbf{P}$.

For internal multiple elimination, the concept of CFP gather is introduced. A CFP gather represents focused data with one source in the subsurface and all receivers at the surface (or, vice versa, for a receiver gather). Thus the prediction of internal multiples will be as same as the prediction of surface-related multiples (Fig. 3).

(a)

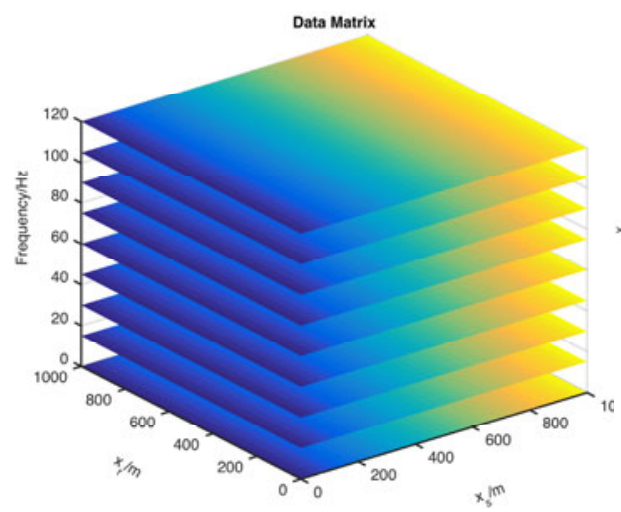

(b)

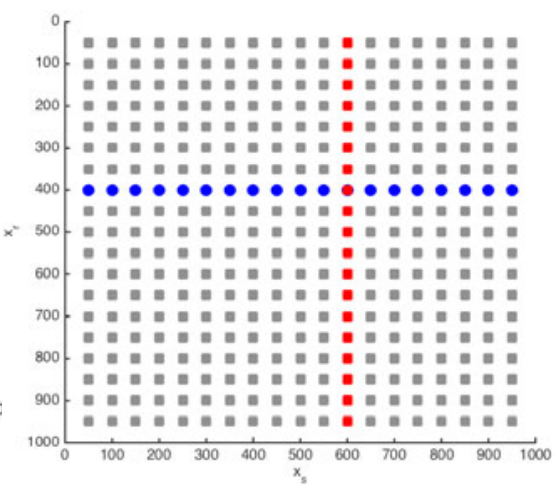

Fig. 1. Data matrices for 2-D seismic data volumes: (a) each slice represents a monochromatic data matrix, (b) in the matrix, the columns contain monochromatic shot records and the rows contain monochromatic common-receiver gathers. 


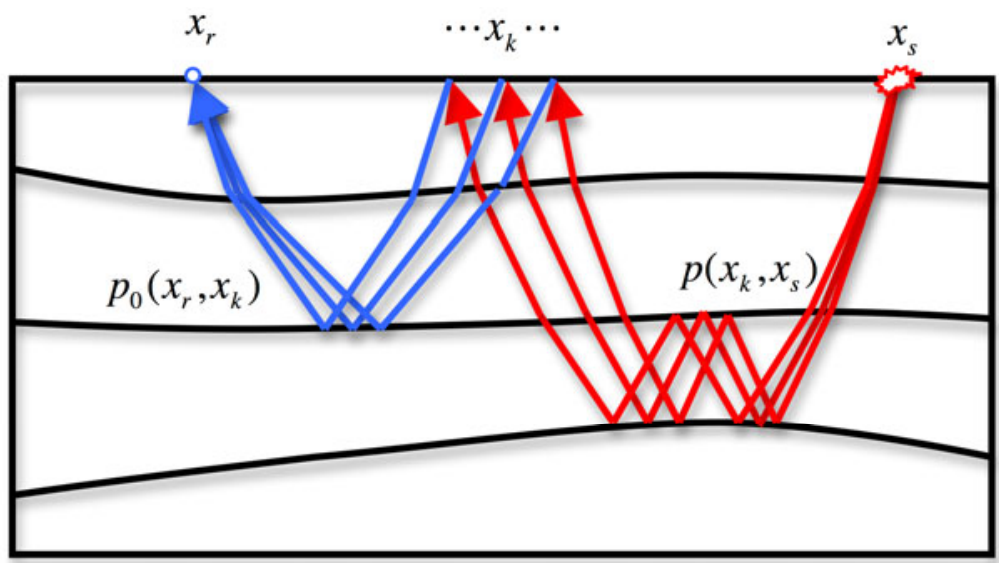

Fig. 2. Surface-related multiple from source $x_{s}$ to receiver $x_{r}$ can be seen as the sum of the convolution of $p_{0}\left(x_{r}, x_{k}\right)$ and $p\left(x_{k}, x_{s}\right)$ for each trace in $t-x$ domain, where $p_{0}\left(x_{r}, x_{k}\right)$ is the data without multiple and $p\left(x_{k}, x_{s}\right)$ is the data with multiple $(k$ is variable).

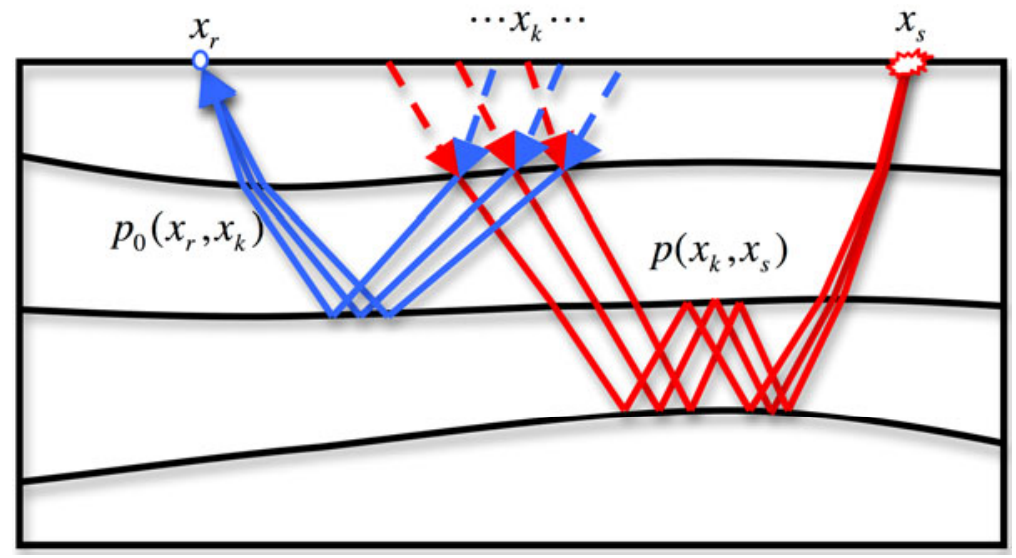

Fig. 3. Internal multiple prediction $p_{0}\left(x_{r}, x_{k}\right)$ and $p\left(x_{k}, x_{s}\right)$ are CFP gathers with sources and receivers at the internal multiple-generating surface, respectively.

Focusing in detection can be formulated as

$$
\mathbf{P}\left(z_{n}, z_{0}\right)=\left[\mathbf{F}\left(z_{n}, z_{0}\right)\right]^{*} \mathbf{P}\left(z_{0}, z_{0}\right) .
$$

In Eq. 3, $z_{0}$ represents the surface and $z_{n}$ represents the internal multiplegenerating surface. $\mathbf{P}\left(z_{n}, z_{0}\right)$ represents the focused gather whose source is at the surface and receivers are at $z_{n}$. $\mathbf{P}\left(z_{0}, z_{0}\right)$ represents the shot gather whose source and receivers are both at the surface. $\mathbf{F}\left(z_{n}, z_{0}\right)$ represents the focusing 


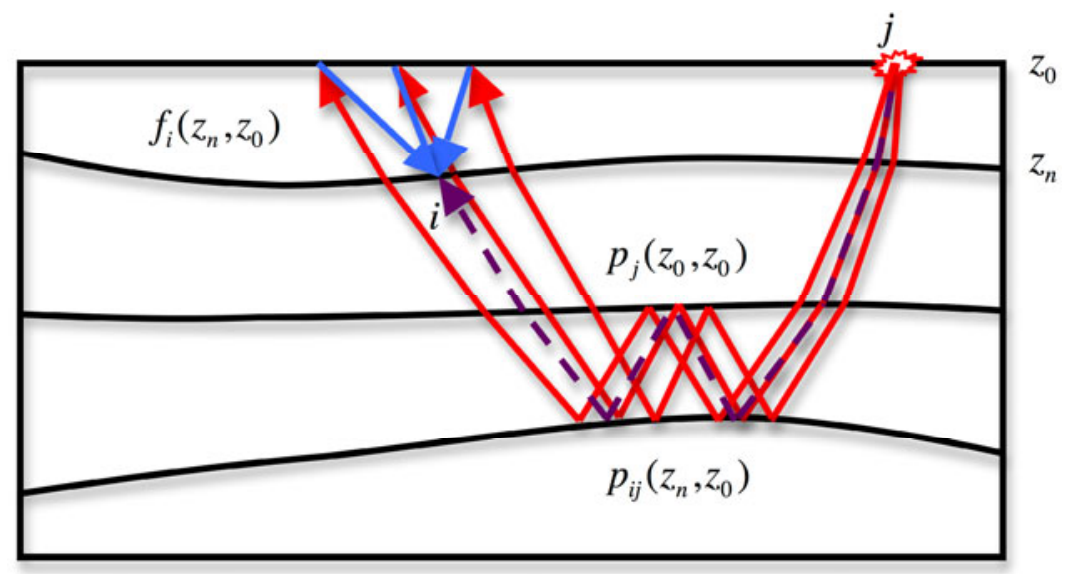

Fig. 4. Construction of one CFP trace for focusing in detection. $p_{\boldsymbol{j}}\left(z_{0}, z_{0}\right)$ is the shot gather with its source positioned at $\left(x_{j}, z_{0}\right)$ and the receivers positioned at the surface. $f_{i}\left(z_{n}, z_{0}\right)$ is the Green's function of the virtual receiver. CFP trace $p_{i j}\left(z_{n}, z_{0}\right)$, whose source positioned at $\left(x_{j}, z_{0}\right)$ and receiver positioned at $\left(x_{i}, z_{n}\right)$, is obtained by summing the time convolution of the shot gather and the time revered Green's function along the spatial axes.

operator. Each row of $\mathbf{F}$ contains the Green's function of the virtual receiver measured at the surface. The focusing process is actually an inverse extrapolation process, thus the time reversed Green's function should be used. The complex conjugate of the Green's function in frequency domain, which is denoted with the superscript $*$ in Eq. 3, stands for time reversal in time domain. In Fig. 4, the process of focusing in receivers is illustrated with ray paths. Similarly, focusing in emission can be formulated as

$$
\mathbf{P}\left(z_{0}, z_{n}\right)=\mathbf{P}\left(z_{0}, z_{0}\right)\left[\mathbf{F}\left(z_{0}, z_{n}\right)\right]^{*}
$$

where $\mathbf{P}\left(z_{0}, z_{n}\right)$ represents the focused gather whose receivers are at the surface and source is at $z_{n}$. Each column of the focusing operator $\mathbf{F}$ contains the Green's function of the virtual source measured at the surface. According to the feedback algorithm, the internal multiples related to boundary $z_{n}$ removal process can be written as

$$
\mathbf{P}_{n}^{(i)}\left(z_{0}, z_{0}\right)=\mathbf{P}_{n-1}\left(z_{0}, z_{0}\right)-A_{n}(f) \overline{\mathbf{P}}_{n}^{(i-1)}\left(z_{0}, z_{n}\right) \overline{\mathbf{P}}_{n-1}\left(z_{n}, z_{0}\right)
$$

In Eq. 5, $\mathbf{P}_{n}\left(z_{0}, z_{0}\right)$ represents the seismic data with all primary reflections and internal multiples for $z>z_{n}$ only. $A_{n}(f)$ is the match filter between the predicted and actual multiples. $\overline{\mathbf{P}}_{n}\left(z_{0}, z_{n}\right)$ represents the seismic data focused in emission with its source positioned at depth level $z_{n}$, with all reflec- 
tions (primaries and multiples) up to level $z_{n}$ removed. $\overline{\mathbf{P}}_{n-1}\left(z_{n}, z_{0}\right)$ represents the seismic data focused in detection with its receivers positioned at depth level $z_{n}$, with all the primaries up to level $z_{n}$ removed and all the multiples up to level $z_{n-1}$ removed. Iteration number is represented by $i$. Internal multiples are generally weaker than surface multiples, so one iteration is often sufficient. Note that the internal multiple elimination should be performed from shallow to deep.

Jakubowicz (1998) proposed the surface-based IME method based on the geometry analysis by Keydar et al. (1997). In the surface-based IME method, CFP gather is not needed anymore; the internal multiples can be estimated directly from measured data at the surface. According to the CFP theory discussed above, we can derive the surface-based IME method based on CFP theory. In this paper, we give a mathematic derivation for surface-based IME method based on CFP theory. According to Eq. 4, the internal multiples are given by

$$
\mathbf{M}_{n}^{(i)}\left(z_{0}, z_{0}\right)=\mathbf{P}_{n}^{(i-1)}\left(z_{0}, z_{n}\right) \mathbf{P}_{n-1}\left(z_{n}, z_{0}\right) .
$$

Using Eqs. 3 and 4, we can also write Eq. 6 as

$$
\mathbf{M}_{n}^{(i)}\left(z_{0}, z_{0}\right)=\overline{\mathbf{P}}_{n}^{(i-1)}\left(z_{0}, z_{0}\right)\left[\Delta \mathbf{P}_{n}\left(z_{0}, z_{0}\right)\right]^{*} \overline{\mathbf{P}}_{n-1}\left(z_{0}, z_{0}\right)
$$

with

$$
\left[\Delta \mathbf{P}_{n}\left(z_{0}, z_{0}\right)\right]^{*}=\left[\mathbf{F}\left(z_{0}, z_{n}\right)\right]^{*}\left[\mathbf{F}\left(z_{n}, z_{0}\right)\right]^{*}
$$

where $\boldsymbol{\Delta} \mathbf{P}_{n}\left(z_{0}, z_{0}\right)$ represents the primary reflection from the multiplegenerating interface $z_{n}$ (the yellow dashed line in Fig. 5). Jakubowicz names $\overline{\mathbf{P}}_{n}^{(i-1)}\left(z_{0}, z_{0}\right), \boldsymbol{\Delta} \mathbf{P}_{n}\left(z_{0}, z_{0}\right)$, and $\overline{\mathbf{P}}_{n-1}\left(z_{0}, z_{0}\right)$ as "source term", "interbed primary", and "response term", respectively. According to the derivation, we can find that the combination of the two focusing operators is equal to the time-reversed reflection of the corresponding reflector. In addition, if we make the "interbed primary" also including the primaries above this boundary, then this method becomes a layer-related version.

Through the theoretical derivation, we proved that the CFP-based and the surface-based IME methods are based on the same core principles but implemented in different forms. Obviously, the surface-based IME method is much more efficient for implementation.

If the approximation of a low-relief structure is valid, the CMP-oriented method can be applied as well. This method is applied on pre-stack data in the CMP domain under the assumption of locally lateral invariance of the earth. This means the multiple prediction process can be carried out on indi- 


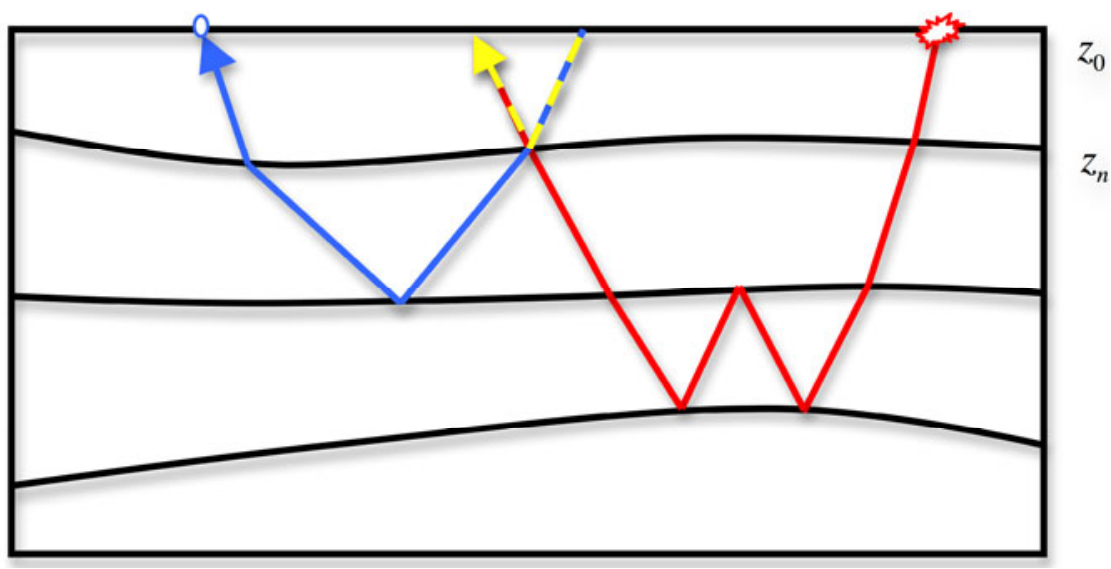

Fig. 5. Internal multiple prediction with the data measured at the surface.

vidual CMP gathers after Fourier transform to the $f-k$ domain. In this case, matrix multiplications in $f-x$ domain reduced to scalar multiplications in $f-k$ domain, and the CMP-oriented internal multiple prediction can be formulated as

$$
M_{n}^{(i)}\left(k_{x}, \omega\right)=\bar{P}_{n}^{(i-1)}\left(k_{x}, \omega\right)\left[\Delta P_{n}\left(k_{x}, \omega\right)\right]^{\#} \bar{P}_{n-1}\left(k_{x}, \omega\right) .
$$

In Eq. $8, \bar{P}_{n}\left(k_{x}, \omega\right)$ represents the seismic data with all primary reflections and internal multiples for $z>z_{n}$ only. $\left[\Delta P_{n}\left(k_{x}, \omega\right)\right]^{\#}$ denotes the time reversed primary reflection of the internal multiple-generating surface in $f-k$ domain. Note that the time-reversed data in $f-k$ domain cannot be directly acquired. Two steps should be taken: first, the complex conjugation of the data is calculated in $f-x$ domain, and then the spatial Fourier transform is performed; this process is denoted with the superscript \#. The CMP-oriented method for internal multiple elimination can be a practical alternative to shot-oriented method, especially when the low-relief structure is valid.

The CMP-oriented method is ideally suited for land data. Land seismic data generally have poor $\mathrm{S} / \mathrm{N}$ ratio; however, the feedback model IME method uses the reflection data itself to predict multiples. That means any nonereflection signals, such as direct wave, surface wave and random noise, will affect the predicted result. Besides, regular offset of the input data is required as well. In CMP domain, a group of neighboring CMP gathers can be merged into a supergather. The CMP supergather has a dense offset distribution and less noise. Offset regulation and trace interpolation could be much easier to perform in a supergather. With the high $\mathrm{S} / \mathrm{N}$ ratio and well-sampled supergather, multiples can be better predicted. 


\section{SYNTHETIC DATA EXAMPLE}

For the demonstration of CMP-oriented internal multiple elimination, both 1D and 2D synthetic data sets are simulated. The time-variant taper based on the first Fresnel zone, which can reduce edge effect more efficiently in the predicted multiples, is proposed and discussed in detail via 1D synthetic data. The 2D anticline structure model is designed to test the effectiveness of the CMP-oriented IME method in a relatively complex structure. In order to focus on the internal multiple elimination, surface multiples are not generated in the synthetic data.

\subsection{D model}

The 1D model is shown in Fig. 6. The CMP gather contains 201 traces in a split-spread configuration with offsets from -500 to $500 \mathrm{~m}$ relative to the source position and the trace interval is $5 \mathrm{~m}$. The synthetic CMP gather is displayed in Fig. 7. Besides the three primaries, internal multiples are clearly visible. The internal multiples have been indicated in the figure by a sequence of bounces (upward-downward-upward, etc.).

Multiple elimination is an iterative process; however, one iteration is sufficient for internal multiple elimination in practice. Thus, only the interbed primary and the response term are required for the prediction (Fig. 8). The two data sets can be obtained directly by applying inside and (or) outside mute to the raw data, but it is more practical to mute the NMO corrected data, and the inverse NMO correction should be applied after the mute.

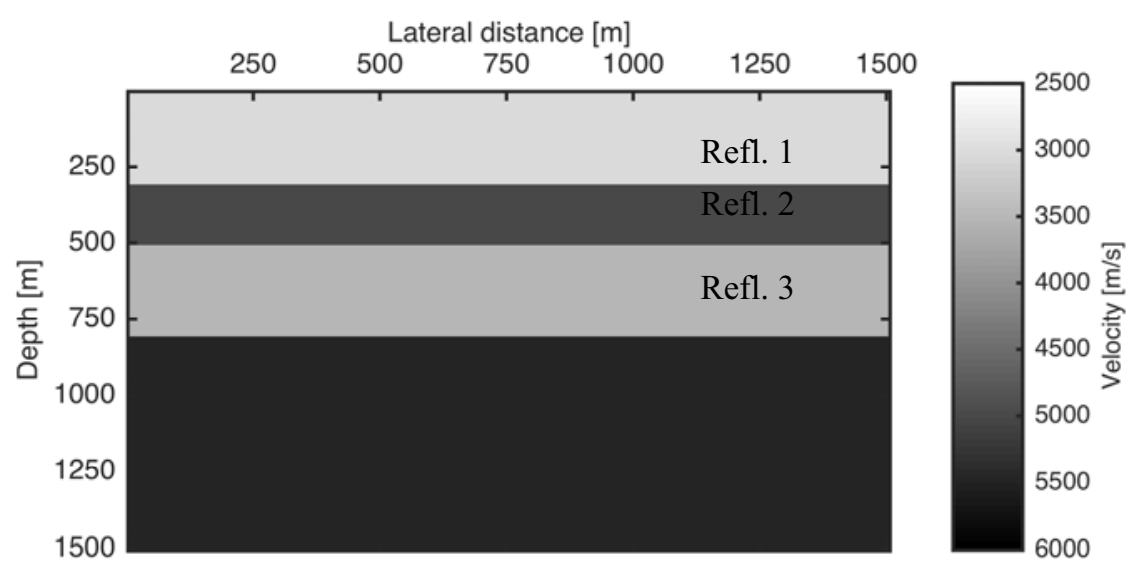

Fig. 6. The 1D subsurface model; a relative high-velocity layer occurs at the depth around 300 to $500 \mathrm{~m}$. 


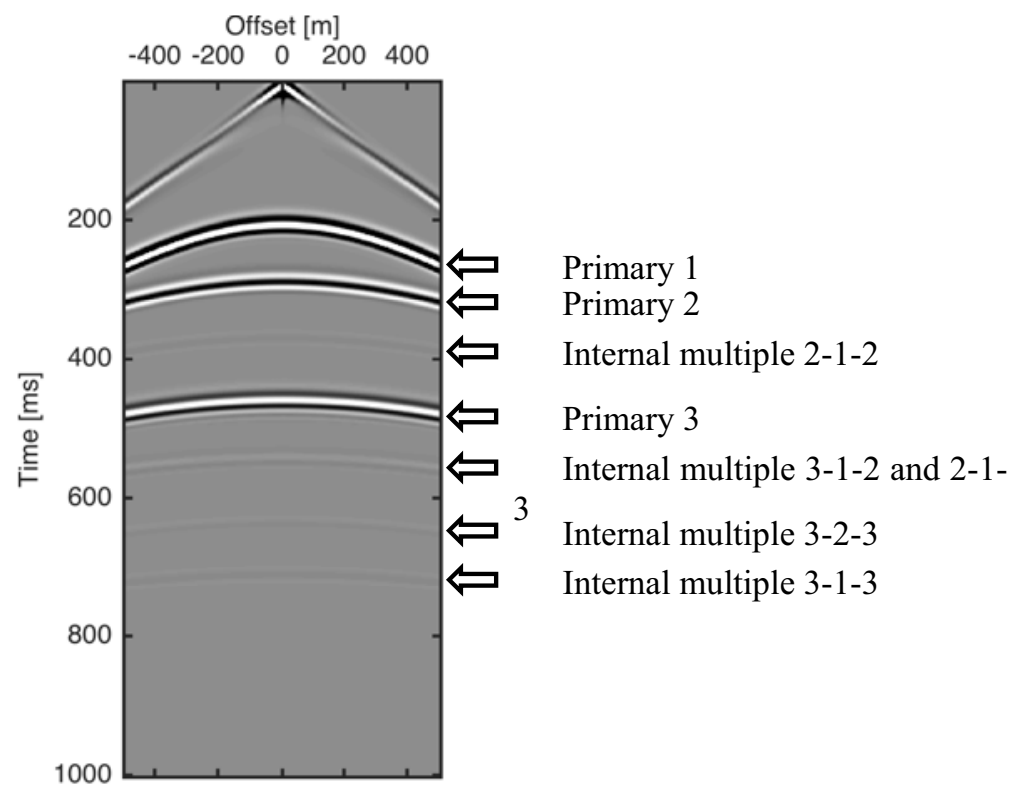

Fig. 7. The synthetic CMP gather.

(a)

(b)
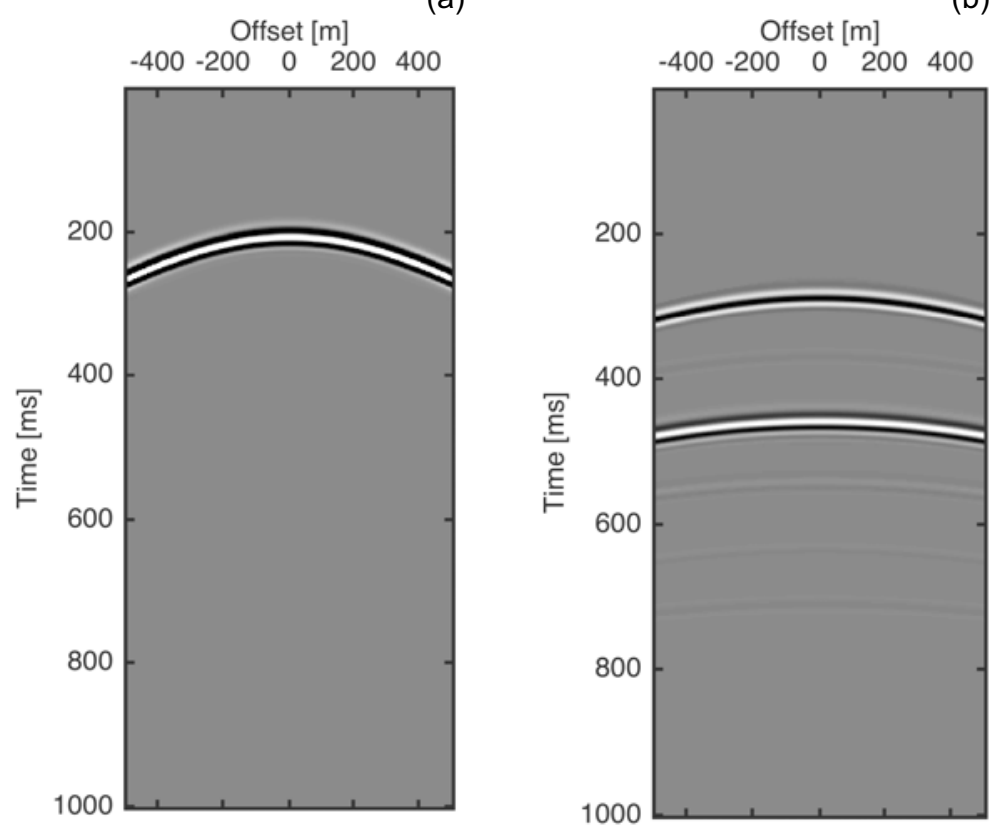

Fig. 8. The wavefield terms used in predicting the internal multiples generated by the first reflector of the data. The interbed primary (a) and response term (b) can be obtained directly by muting the initial data. 
Multiple elimination involves prediction and subtraction. First, apply multiple prediction by multiplying the prepared data in the $f-k$ domain. In practical prediction, the limited aperture of the data may cause edge effects in the predicted multiples, with genuine multiple events appearing to reflect back into the data (Fig. 9a). The first Fresnel zone plays an important role in multiple prediction; however, the energy outside the first Fresnel zone will actually introduce artifacts in the predicted results when the aperture is limited. Thus, tapering the amplitudes outside the first Fresnel zone would be an efficient way to reduce these edge effects. Generally, the radius of the first Fresnel zone will increase as the depth goes deeper, so a time variant taper would be more proper for the reduction of the edge effects. In this paper, a time variant taper function is designed as follows:

$$
\left\{\begin{array}{l}
w=1, x \leq R(t) \\
w=\cos \left(\frac{\pi}{2}\left(\frac{x-R(t)}{x_{\max }-R(t)}\right)\right)^{2}, x>R(t)
\end{array}\right.
$$

with

$$
R(t)=\sqrt{\left(v_{t} t+\frac{v_{t}}{4 f}\right)^{2}-\left(v_{t} t\right)^{2}}
$$

where $w$ represents the weighting coefficient of the sample point positioned at time $t$ and offset $x . R(t)$ represents the radius of the First Fresnel zone at time $t . x_{\max }$ is the max offset distance in the gather. We can estimate the approximate radius of the first Fresnel zone from Eq. 9a. In Eq. 9a, $v_{t}$ represents the root mean square velocity at time $t, f$ represents the main frequency of the seismic data. Only approximate velocity and frequency are required as the results are not very sensitive to these parameters. Figure $9 \mathrm{~b}$ shows the predicted multiples with the use of a constant taper. The edge effects are reduced significantly; however, the multiples at far offset become weak, especially at greater depths. With the use of the time variant taper, the energy of the multiples at far offset is preserved (Fig. 9c).

The next step is to subtract the predicted multiples from the input data. As amplitude and phase errors often existed in the predicted multiples, a two-step adaptive subtraction (Verschuur and Berkhout 1997) is applied in this procedure. First, estimate a long filter (i.e., typical 21 to 31 points) for optimization of the whole gather, and then use that result for a second adaptation step within local time and/or offset windows with smaller filters (i.e., typicaly 5 points). The result of this subtraction is shown in Fig. 10: the input CMP gather with internal multiples (Fig. 10a), the result after multiple removal (Fig. 10b), and the estimated internal multiples (Fig. 10c). 
(a)

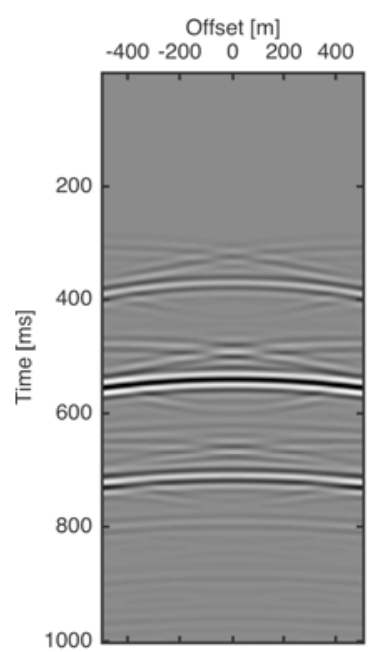

(b)

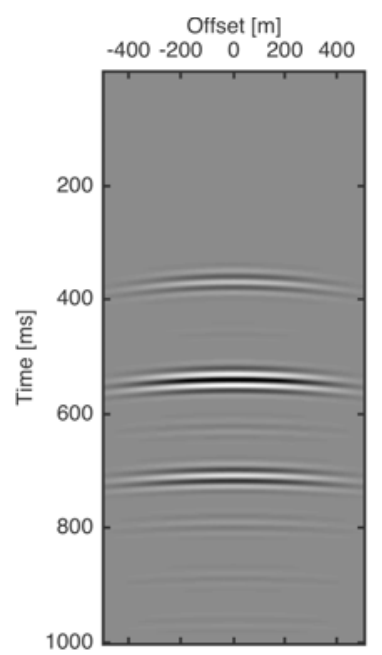

(c)

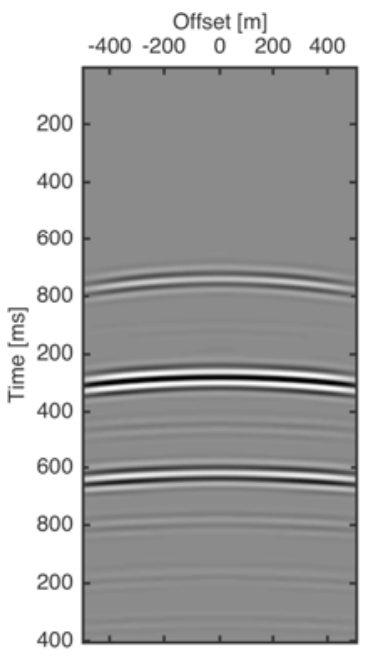

Fig. 9. The predicted multiples (a) are affected by the edge effects severely. With the use of a constant taper, the edge effects in the predicted multiples (b) are reduced significantly, whereas the multiples at far offset are also reduced. Using the time variant taper can preserve the energy of multiples at far offset (c).

(a)

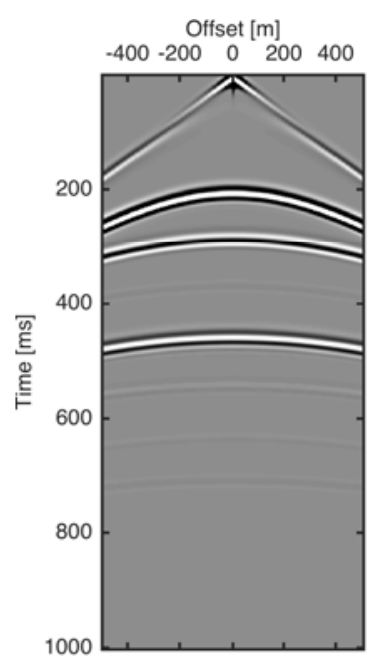

(b)

(c)
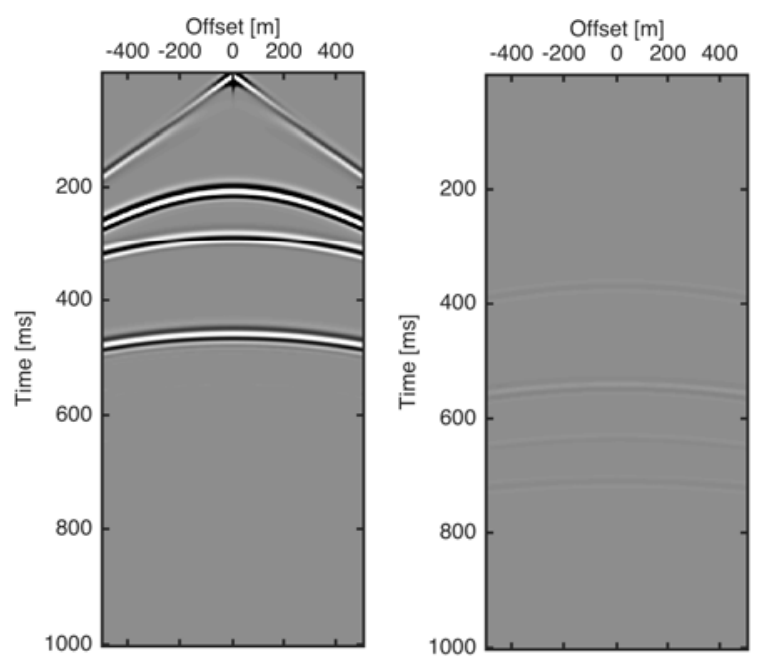

Fig. 10. CMP-oriented internal multiple removal for the 1D data set: (a) CMP gather with internal multiples, (b) CMP gather after internal multiple elimination, (c) internal multiples removed. 


\subsection{D model}

The anticline structure model, shown in Fig. 11, is designed to test the effectiveness of the CMP-oriented method in 2D situation. The same procedure is applied on this data set. Figure 12 shows the multiple elimination results of 3

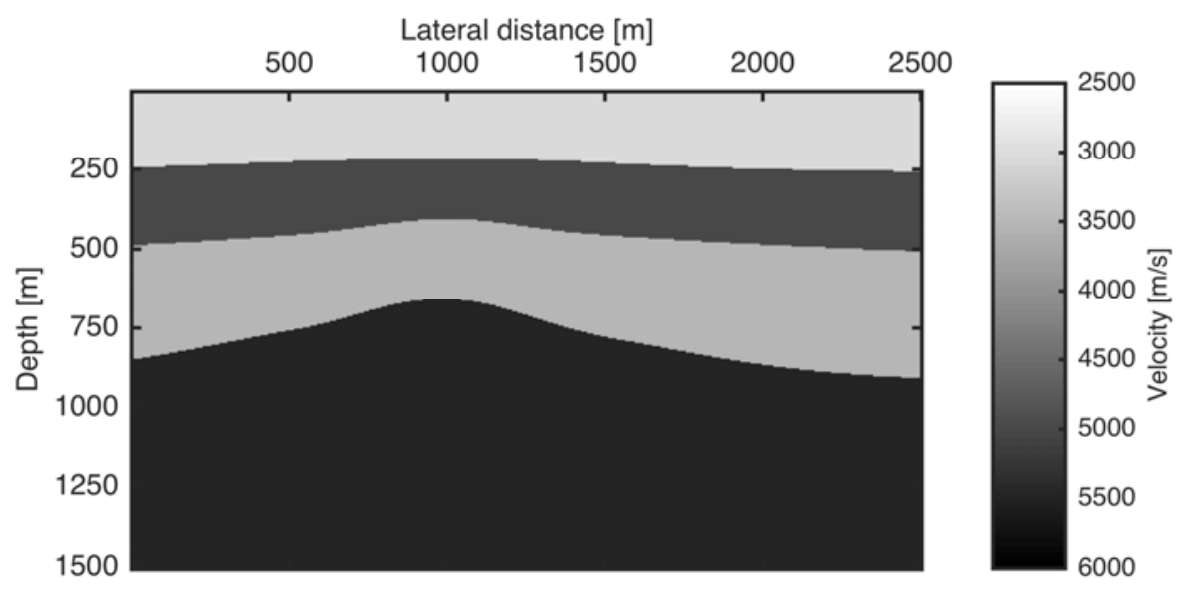

Fig. 11. The anticline structure model.

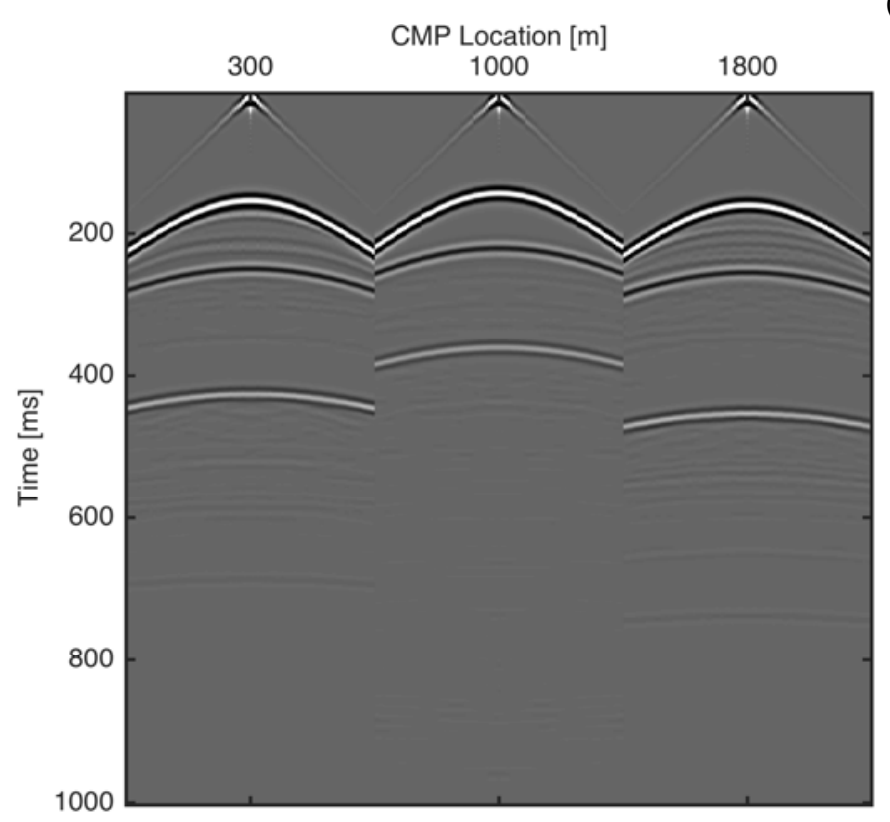

(a)

Fig. 12. Continued on next page. 
(b)

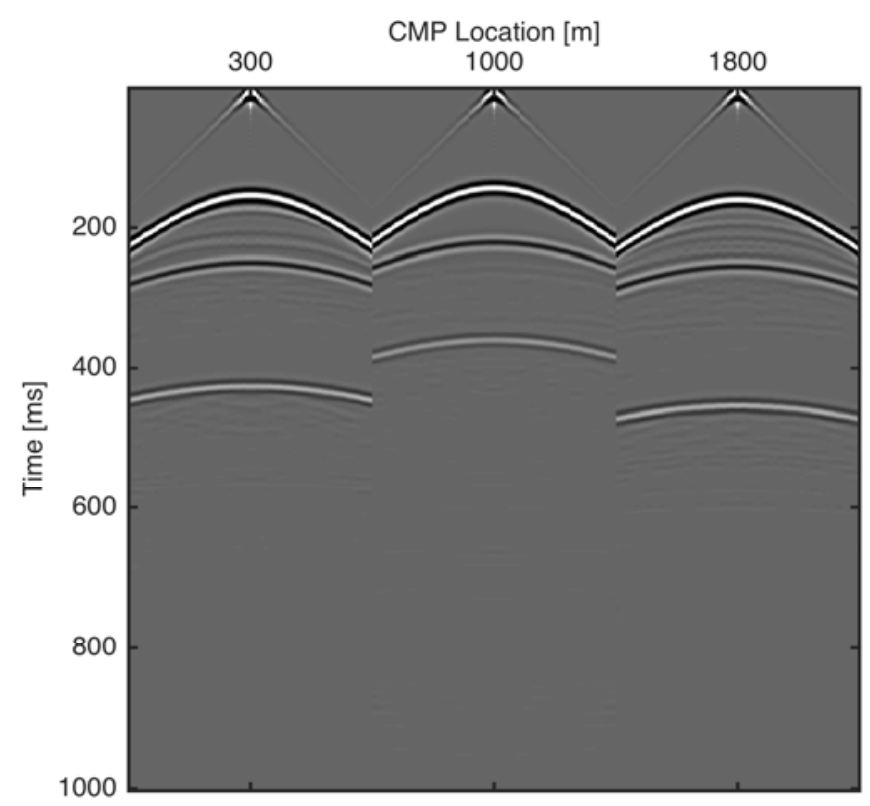

(c)

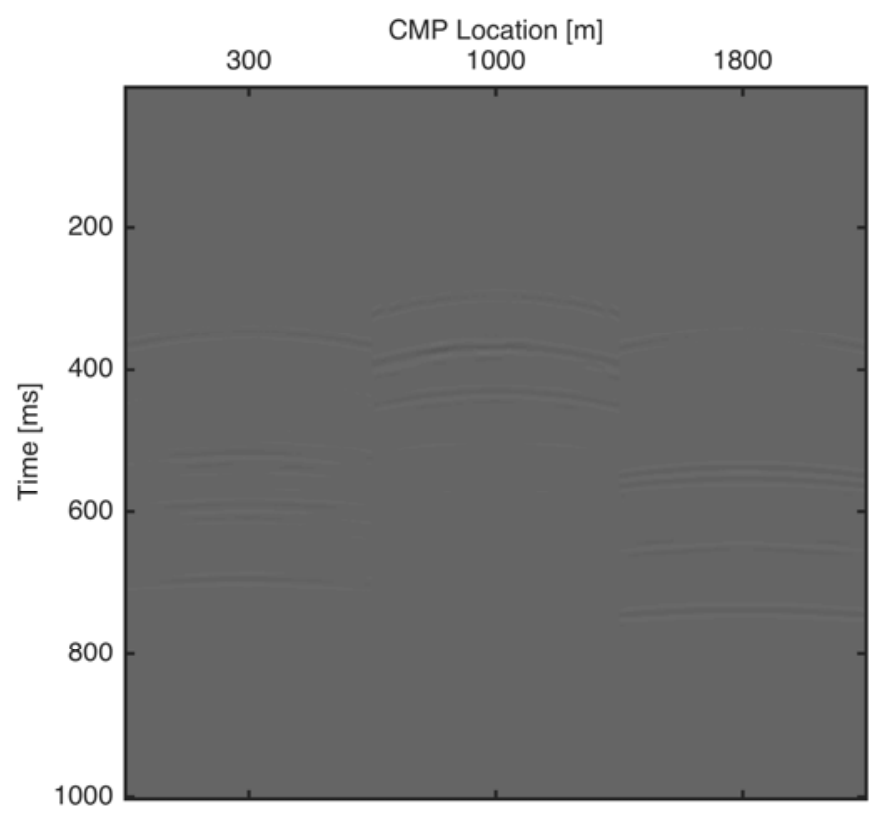

Fig. 12. CMP gathers at different locations, before (a) and after (b) internal multiple attenuation, and the adaptively subtracted internal multiples (c). 


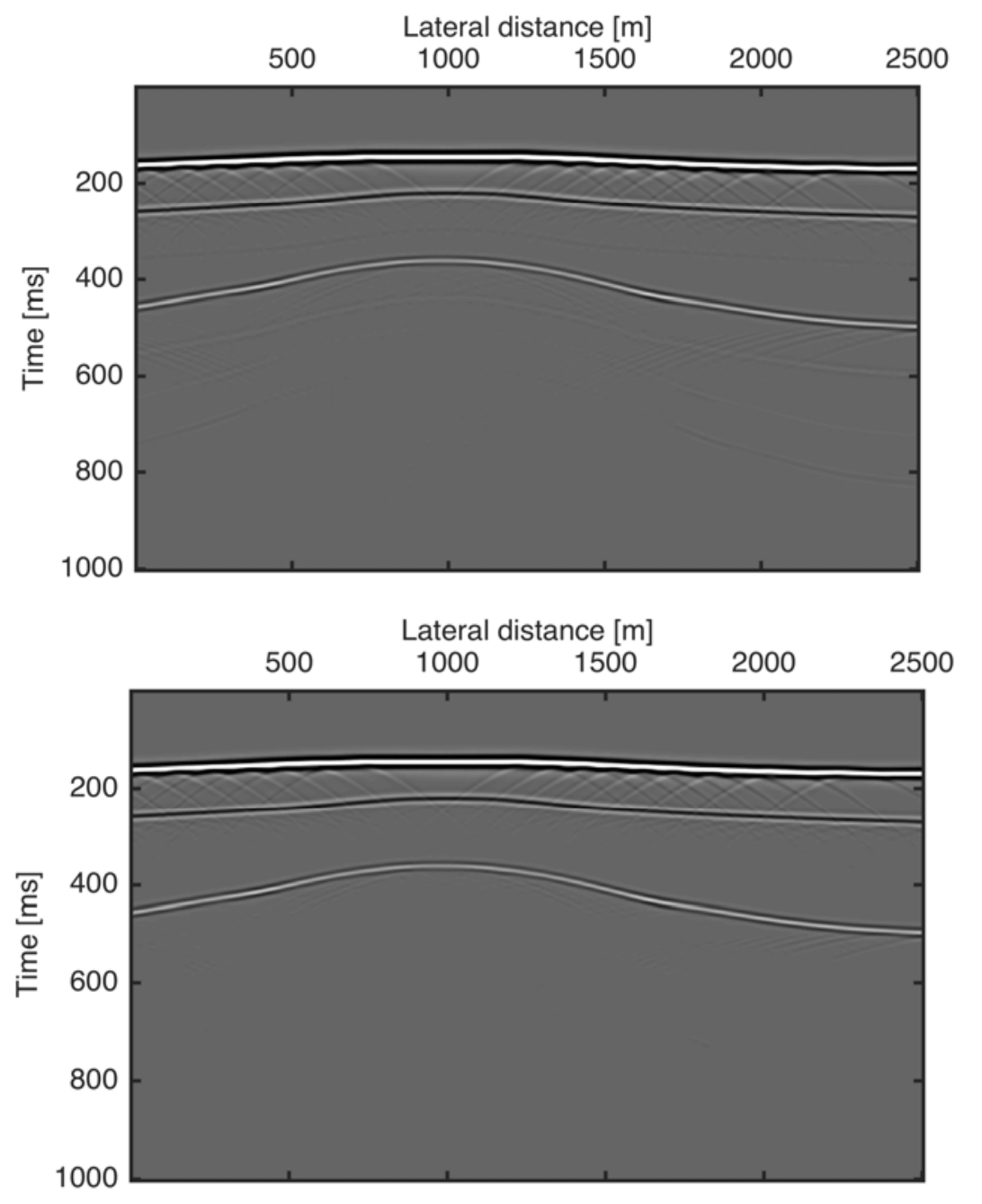

(a)

(b)

Fig. 13. Stack results before (a) and after (b) the internal multiple elimination.

CMP gathers at different locations. Note that the diffractions have also existed in the input data (Fig. 12a). The CMP gathers after multiple removal is shown in Fig. 12b, and the removed multiples are shown in Fig. 12c. After applying the CMP-oriented internal multiple suppression, the CMP gathers are stacked (Fig. 13). The internal multiples can be observed in the input stacked section (Fig. 13a) clearly. Figure 13b shows the primaries-only stack; the internal multiples are cleanly removed. From this example, we can see the CMP-oriented method can still produce good results when the medium is not totally $1 \mathrm{D}$. 


\section{FIELD DATA EXAMPLE}

When dealing with the land seismic data, internal multiple elimination becomes much more difficult. Low $\mathrm{S} / \mathrm{N}$ ratio, irregular offset and missing traces impose additional constrains in the prediction of multiples. Some key techniques could be applied to solve these problems effectively in the CMP domain; therefore, the CMP-oriented IME method would be more appropriate for land seismic data. The detailed procedure of preprocessing is demonstrated as follows. In addition, surface-related multiples are proved to be not existing in the filed land seismic data we used in this paper via SRME method.

Traditional preprocessing steps include static correction, noise reduction and offset regulation. High frequency static corrections should be applied to make the reflections more coherent. Ground roll could be suppressed with an $f-k$ filter. Linear noise, such as refractions, can be removed with the radial trace filter. Then the CMP gathers are regularized via NMO correction and trace repositioning. The CMP gather after traditional preprocessing is shown in Fig. 14a, but it is still not good enough to obtain a satisfactory result. By merging a group of neighboring CMP gathers (in this case 7) to one supergather, a dense offset distribution is simulated and the $\mathrm{S} / \mathrm{N}$ ratio of the gather is improved as well (Fig. 14b). For more complex structures, a smaller number of neighboring CMP gathers, such as 5 or 3, is recommended; for merging the supergather, we should balance the tradeoff between noise attenuation and detail preservation. Interpolation is performed after applying an approximate NMO correction to the supergather, and then we apply a lateral smoothing to reduce the random noise. At last, an inverse NMO correction is applied. Figure 14c shows the well prepared CMP supergather for the multiple prediction.

In addition, before the multiple prediction, the deep reflection energy of the response term should be attenuated to avoid the extra predicted multiples emerging at the top of the predicted results due to the limitation of the acquisition time (Fig. 15).

Note that, in this field data, weak reflectors (above $1000 \mathrm{~ms}$ ) did not induce significant internal multiples; several strong reflectors generated most of the internal multiples. Thus, even though the layer-related version can predict the multiples related to more boundaries at one time, taking all boundaries into consideration in the multiple prediction process is unnecessary and sometime it could even decrease the effectiveness of the adaptive subtraction because the adaptive filter has to take care of both significant multiples generated by strong reflectors and insignificant multiples generated by weak reflectors at the same time. In this situation, the boundary-related version is more targeted to remove the specific internals generated by a cer- 
(a)

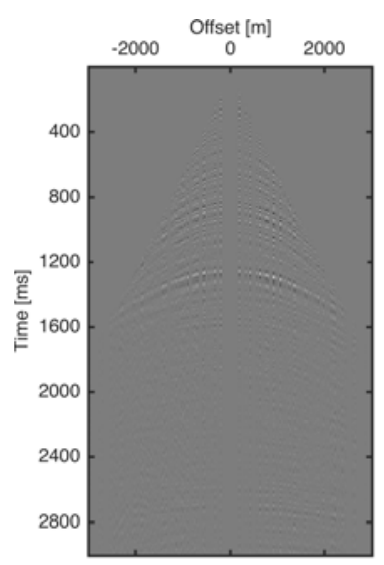

(b)

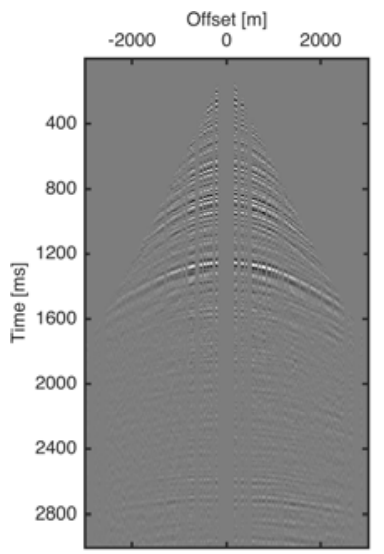

(c)

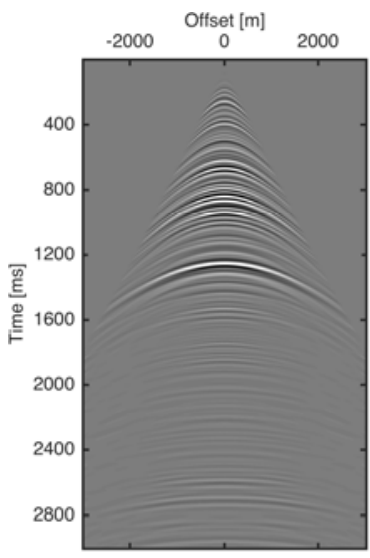

Fig. 14: (a) A CMP gather after regular preprocessing, plotted on true offset; (b) a CMP supergather after merging 7 CMP gathers; (c) supergather after interpolation and random noise reduction.

(a)

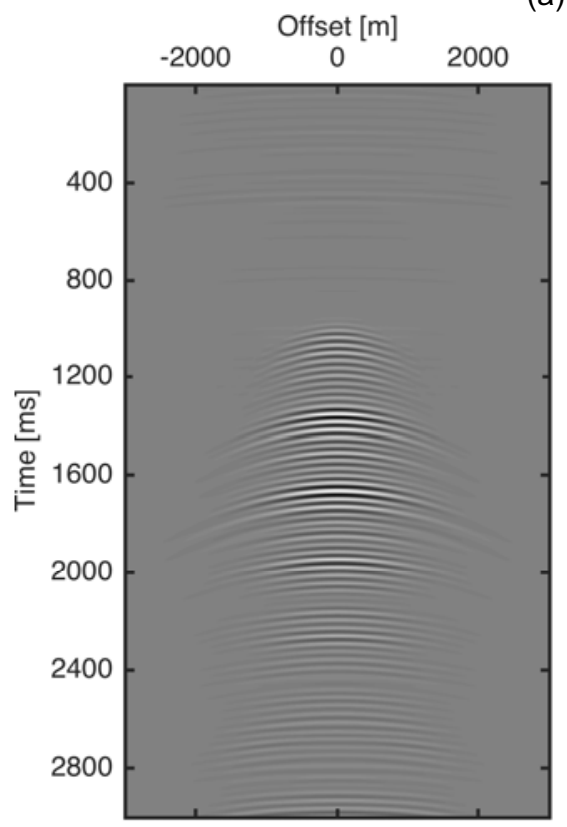

(b)

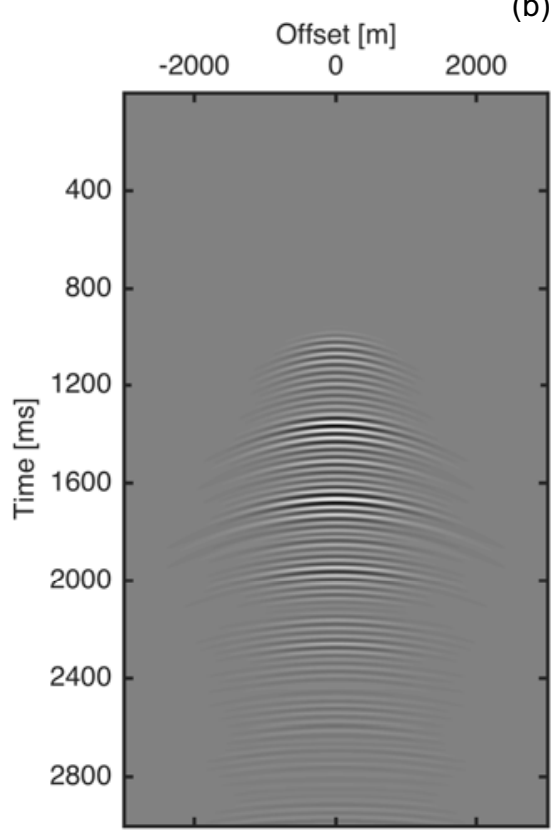

Fig. 15. Predicted results with extra multiples at the top (a), after applying the attenuation, the predicted multiples are free from the interference of the extra multiples (b). 
(a)

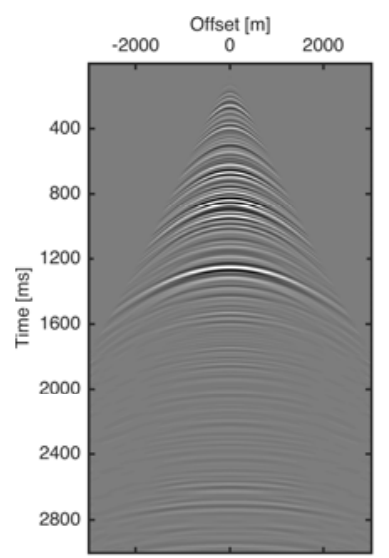

(b)

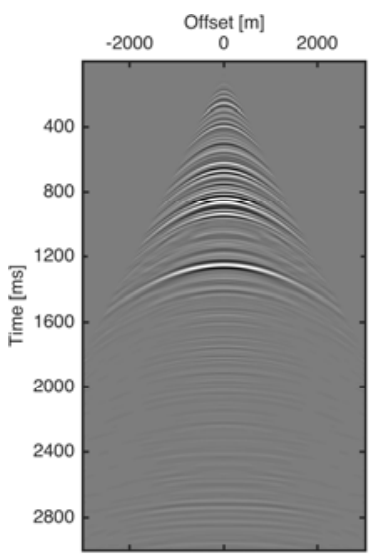

(c)

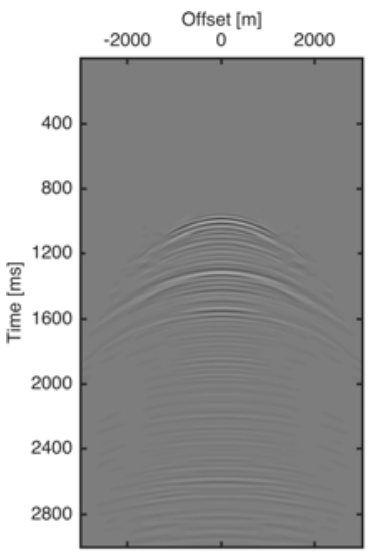

Fig. 16. CMP-oriented multiple removal for land seismic data: (a) CMP gather with multiples; (b) CMP gather after internal multiple suppression; (c) internal multiples removed.

(a)

(b)
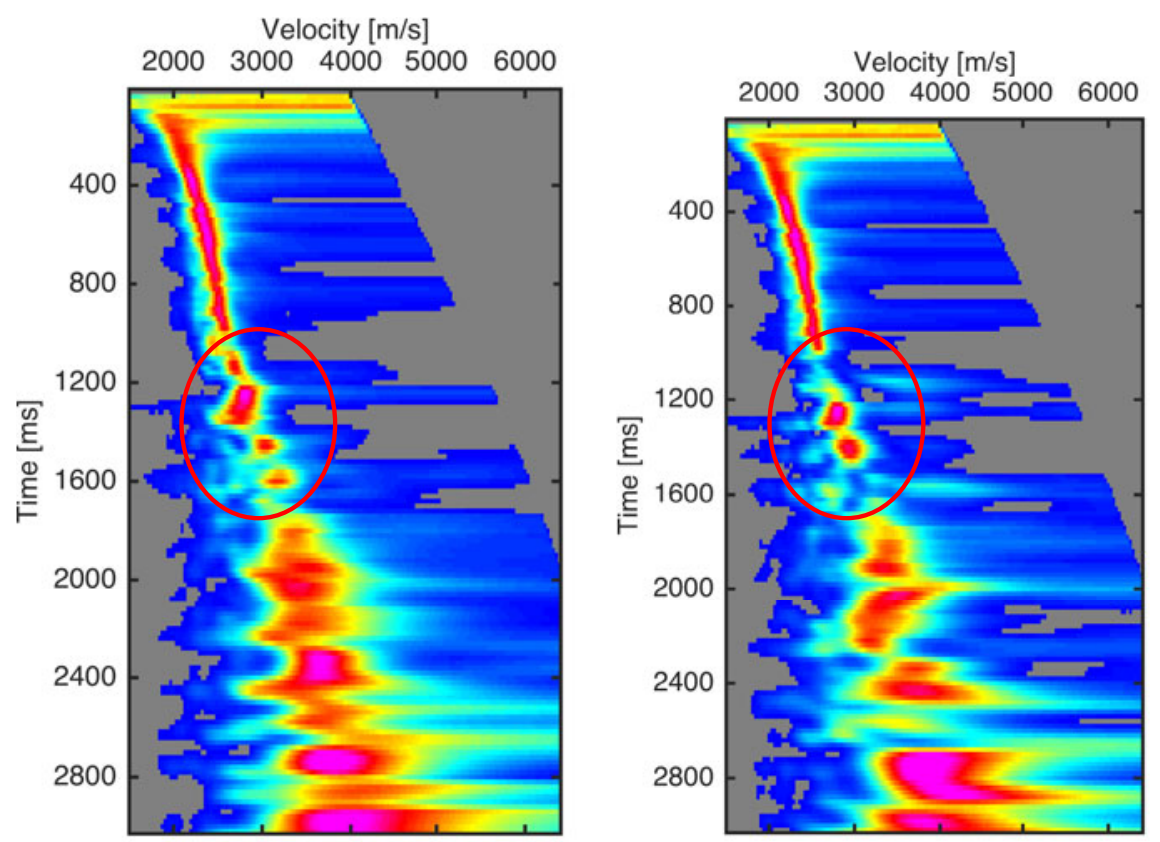

Fig. 17. The velocity spectrums of the CMP gathers before (a) and after (b) internal multiple elimination. 
(a)

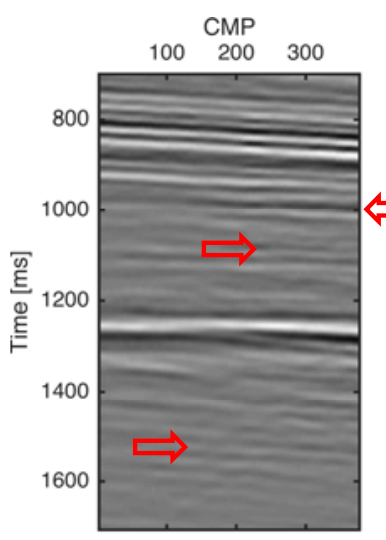

(d)

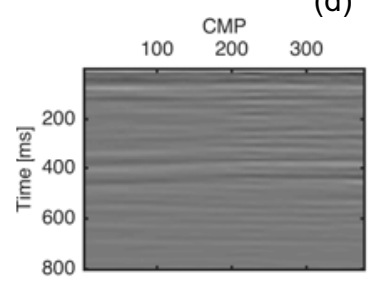

(b)

(c)

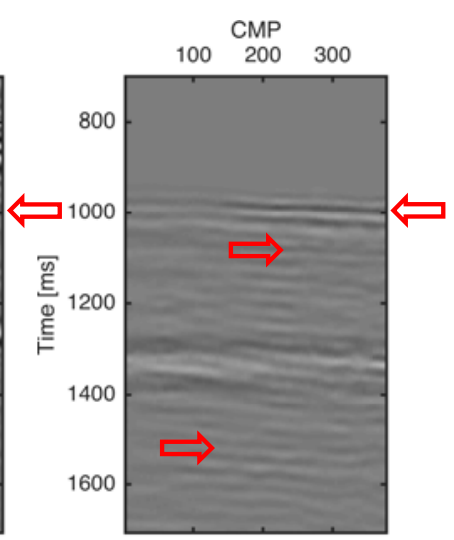

(e)

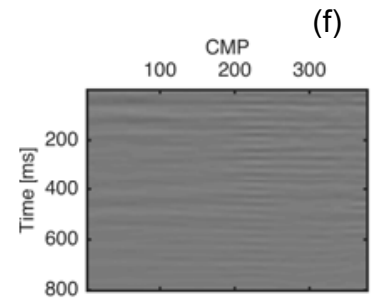

Fig. 18. Stacked land section before (a) and after (b) internal multiple elimination, and (c) the adaptively subtracted internal multiples. The autocorrelations of these stack results are shown in (d), (e), and (f), respectively.

tain strong reflector. In this example, three strong internal multiplegenerating surfaces are selected to predict the internal multiples. The result of the internal multiple elimination is shown in Fig. 16, and the velocity spectrums of two CMP gathers (before and after multiple elimination) are shown in Fig. 17. The relatively low velocity energy, for example the energy occurred at 1100 and $1300 \mathrm{~ms}$ in Fig. 17a, generally implies the existence of internal multiples, and, sometimes, internal multiples also experience similar (or even higher) velocities to primaries in their vicinity, such as the energy occurred at $1600 \mathrm{~ms}$. In Fig. 17b, the multiple energy is significantly removed, thus reducing a lot of the picking ambiguity. Figure 18a shows a local stack result of this land data set before internal multiple elimination. Several strong reflectors, which occurred at 850 and $1250 \mathrm{~ms}$, particularly induced the generation of the internal multiples. The autocorrelation of this stack section is shown in Fig. 18d, periodic energy implies the existence of the multiples. The primaries-only stack is shown in Fig. 18b, the internal 
multiples are significantly removed. For the convienience of comparison, red arrows are added to the stack sections (Fig. 18). Periodic energy is apparently reduced in its autocorrelation, as shown in Fig. 18e. Figure 18c shows the removed internal multiples, and its autocorrelation is shown in Fig. 18f.

\section{CONCLUSIONS}

We have successfully extended the data-driven IME method to the CMP domain. For low-relief structures, the CMP-oriented method is more suitable for land seismic data with more challenging problems than marine data.

Proper preprocessing of data is essential in order to obtain satisfactory results. The raw data should be preprocessed with high-frequency static corrections, noise reduction, offset regulation, CMP supergather composition, interpolation and random noise attenuation sequentially to improve the $\mathrm{S} / \mathrm{N}$ ratio and densify the offset distribution. When the predicted results are plagued with edge effects, attenuating the far offset energy with the time variant taper will effectively reduce the edge effects, without attenuating the predicted multiples. In addition, the multiples caused by deep reflectors may occur at the top of the predicted results due to the limitation of acquisition time, and this could be avoided by simply attenuating the deep reflection energy of the response term. These techniques improved the accuracy of the predicted multiples and therefore the multiples can be adaptively subtracted more effectively. The boundary-related method is more suitable and targeted for internal multiple elimination of the seismic data most of whose internal multiples are generated only by several strong reflectors. The field data example shows that satisfactory results can be obtained by just taking several strong subsurface reflectors into consideration.

The CMP-oriented IME method is applied to synthetic data sets and a land field data set; the application examples demonstrate the effectiveness of the proposed methodology.

Acknowledgments. We greatly appreciated the constructive suggestions from editors and anonymous reviewers. This work is supported by the Geologic Survey Project of China (Grant No. DD20160161), and the Fundamental Research Funds for the Central Universities of Chian (Grant No. YYWF201506). 


\section{References}

Alá'i, R., and D.J. Verschuur (2006), Case study of surface-ralated and internal multiple elimination on land data. In: 76th Annual International Meeting, SEG, Expanded abstracts.

Araujo, F.V., A.B. Weglein, P.M. Carvalho, and R.H. Stolt (1994), Inverse scattering series for multiple attenuation: An example with surface and internal multiples. In: 64th Annual International Meeting, SEG, Expanded abstracts, 1039-1041.

Berkhout, A.J. (1982), Seismic Migration: Imaging of Acoustic Energy by Wavefield Extrapolation. A. Theoretical Aspects, 2nd ed., Elsevier Science Publ. Co., Amsterdam.

Berkhout, A.J. (1997), Pushing the limits of seismic imaging. Part I: Prestack migration in terms of double dynamic focusing, Geophysics 62, 3, 937-953, DOI: 10.1190/1.1444201.

Berkhout, A.J., and D.J. Verschuur (1997), Estimation of multiple scattering by iterative inversion. Part I: Theoretical considerations, Geophysics 62, 5, 1586-1595, DOI: 10.1190/1.1444261.

Berkhout, A.J., and D.J. Verschuur (2005), Removal of internal multiples with the common-focus- point (CFP) approach - Part 1: Explanation of the theory, Geophysics 70, 3, V45-V60, DOI: 10.1190/1.1925753.

Coates, R.T., and A.B.Weglein (1996), Internal multiple attenuation using inverse scattering: Results from prestack 1 and 2D acustic and elastic synthetics. In: 66th Annual International Meeting, SEG, Expanded abstracts, 15221525.

Jakubowicz, H. (1998), Wave equation prediction and removal of interbed multiples. In: 68th Annual International Meeting, SEG, Expanded abstracts, 15271530, DOI: 10.3997/2214-4609.201408173.

Kelamis, P.G., D.J. Verschuur, K.E. Erickson, R.L. Clark, and R.M. Burnstad (2002), Data-driven internal multiple-attenuation applications and issues on land data. In: 72nd Annual International Meeting, SEG, Expanded abstracts, 2035-2038, DOI: 10.1190/1.1817099.

Kelamis, P.G., W.H. Zhu, K.O. Rufaii, and Y. Luo (2006), Land multiple attenuation - the future is bright. In: 76th Annual International Meeting, SEG, Expanded abstracts.

Keydar, S., E. Landa, B. Gurevich, and B. Gelchinsky (1997), Multiple prediction using wavefront characteristics of primary reflections, In: 59th Conference, EAGE, Geneva, Switzerland, Extended abstracts, Paper A016.

Luo, Y., P.G. Kelamis, Q. Fu, S.G. Huo, G. Sindi, S. Aramco, S.Y. Hsu, and A.B. Weglein (2011), Elimination of land internal multiples based on the inverse scattering series, The Leading Edge 30, 8, 884-889, DOI: 10.1190/ 1.3626496 . 
Song, J.W., E.Verschuur, and X.Chen (2013), Comparing three feedback internal multiple elimination methods, J. Appl. Geophys. 95, 66-76, DOI: 10.1016/ j.jappgeo.2013.05.010

Thorbecke, J.W. (1997), Common focus point technology, Ph.D. Thesis, Delft University of Technology, Delft, The Netherlands.

van Borselen, R.G. (2002), Fast-track, Data-driven interbed multiple removal - a North Sea data example. In: 64th EAGE Conference and Exhibition 2002, Extended abstracts.

van Groenestijn, G.J.A., and D.J. Verschuur (2009a), Estimating primaries by sparse inversion and application to near-offset data reconstruction, Geophysics 74, 3, A23-A28, DOI: 10.1190/1.3111115.

van Groenestijn, G.J.A., and D.J. Verschuur (2009b), Estimation of primaries and near offsets by sparse inversion: marine data applications, Geophysics 74, 6, R119-R128, DOI: 10.1190/1.3213532.

Verschuur, D.J. (1991), Surface-related multiple elimination, an inversion approach, Ph.D. Thesis, Delft University of Technology, Delft, The Netherlands.

Verschuur, D.J., and A.J. Berkhout (1997), Estimation of multiple scattering by iterative inversion. Part II: Practical aspects and examples, Geophysics 62, 5, 1596-1611, DOI: 10.1190/1.1444262.

Verschuur, D.J., and A.J. Berkhout (2005), Removal of multiples with the commonfocus-point (CFP) approach - Part 2: Application strategies and data examples, Geophysics 70, 3, V61-V72, DOI: 10.1190/1.1925754.

Verschuur, D.J., and R.J. Prein (1999), Multiple removal results from Delft University, The Leading Edge 18, 1, 86-91, DOI: 10.1190/1.1438164.

Verschuur, D.J., A.J. Berkhout, and C. Wapenarr (1992), Adaptive surface-related multiple elimination, Geophysics 57, 9, 1166-1177, DOI: 10.1190/ 1.1443330 .

Weglein, A.B. (1999), Multiple attenuation: an overview of recent advances and the road ahead, The Leading Edge 18, 1, 40-44, DOI: 10.1190/1.1438150.

Weglein, A.B., F. Gasparotto, P. Carvalho, and R.H. Stolt (1997), An inversescattering series method for attenuating multiples in seismic reflection data, Geophysics 62, 6, 1975-1989, DOI: 10.1190/1.1444298.

Ypma, F.H.C., and D.J.Verschuur (2013), Estimating primaries by sparse inversion, a generalized approach, Geophys. Prospect. 61, s1, 94-108, DOI: 10.1111/ j.1365-2478.2012.01095.X

Yuan, Y., D. Han, and R. Zhang (2009), Multiple suppression on land seismic data case history. In: 79th Annual International Meeting, SEG, Expanded abstracts, The Society of Exploration Geophysicists, Tulsa, USA, 3093-3097.

Received 16 November 2015

Received in revised form 1 March 2016

Accepted 26 July 2016 